\title{
Intracellular Organization of Insulin Signaling and GLUT4 Translocation
}

\author{
Robert T. Watson and Jeffrey E. Pessin \\ Department of Physiology \& Biophysics, 51 Newton Road, \\ The University of Iowa, Iowa City, Iowa 52242
}

\begin{abstract}
Glucose is cleared from the bloodstream by a family of facilitative transporters (GLUTs), which catalyze the transport of glucose down its concentration gradient and into cells of target tissues, primarily striated muscle and adipose. Currently, there are five established functional facilitative glucose transporter isoforms (GLUT1-4 and GLUTXI), with GLUT5 being a fructose transporter. GLUT1 is ubiquitously expressed with particularly high levels in human erythrocytes and in the endothelial cells lining the blood vessels of the brain. GLUT3 is expressed primarily in neurons and, together, GLUT1 and GLUT3 allow glucose to cross the blood-brain barrier and enter neurons. GLUT2 is a low-affinity (high $\mathrm{Km}$ ) glucose transporter present in liver, intestine, kidney, and pancreatic $\beta$ cells. This transporter functions as part of the glucose sensor system in $\beta$ cells and in the basolateral transport of intestinal epithelial cells that absorb glucose from the diet. A new facilitative glucose transporter protein, GLUTX1, has been identified and appears to be important in early blastocyst development. The GLUT4 isoform is the major insulin-responsive transporter that is predominantly restricted to striated muscle and adipose tissue. In contrast to the other GLUT isoforms, which are primarily localized to the cell surface membrane, GLUT4 transporter proteins are sequestered into specialized storage vesicles that remain within the cell's interior under basal conditions. As postprandial glucose levels rise, the subsequent increase in circulating insulin activates intracellular signaling cascades that ultimately result in the translocation of the GLUT4 storage compartments to the plasma membrane. Importantly, this process is readily reversible such that when circulating insulin levels decline, GLUT4 transporters are removed from the plasma membrane by endocytosis and are recycled back to their intracellular storagc compartments. Therefore, by establishing an internal membrane compartment as the default localization for the GLUT4 transporters, insulin-responsive tissues are poised to respond rapidly and efficiently to fluctuations in circulating insulin levels. Unfortunately, the complexity of these regulatory processes provides numerous potential targets that may be defective and eventually result in peripheral tissue insulin resistance and possibly diabetes. As such, understanding the molecular details of GLUT4 expression, GLUT4 vesicle compartment biogenesis, GLUT4 sequestration, vesicle trafficking, and fusion with the plasma membrane has become a major focus for many laboratories. This chapter will focus on recently elucidated insulin signal transduction pathways and GLUT4 vesicle trafficking components that are necessary for insulin-stimulated glucose uptake and GLUT4 translocation in adipoctyes.
\end{abstract}




\section{Proximal Insulin Receptor Signaling}

The mature cell surface insulin receptor is composed of two extracellular $\alpha$ and two transmembrane $\beta$ subunits disulfide-linked into an $\alpha_{2} \beta_{2}$ heterotetrameric structure (Czech and Corvera, 1999; Lee and Pilch, 1994). Following insulin binding to the extracellular subunits, a transmembrane conformational change is generated that activates the intracellular subunit tyrosine kinase domain. Subsequently, the $\beta$ subunits undergo a series of intramolecular transautophosphorylation reactions, resulting in tyrosine autophosphorylation at multiple sites. For example, tyrosine phosphorylation at the juxtamembrane Y960 residue is necessary for appropriate substrate recognition, whereas tyrosine phosphorylation at residues $\mathrm{Y} 1146, \mathrm{Y} 1150$, and $\mathrm{Y} 1151$ in the kinase activation domain relieves pseudosubstrate inhibition and results in the constitutive activation of insulin receptor substrate protein tyrosine kinase activity.

However, in contrast to many other receptor tyrosine kinases that directly recruit effector molecules to the phosphorylated receptor, the insulin receptor phosphorylates several proximal intracellular targets that serve as docking sites for effector proteins. These include the four members of the insulin receptor substrate family (i.e., IRSI, 2, 3, and 4), Gabl, Shc, signal-regulated proteins (SIRPs), Cbl, and the newly identified APS (adapter protein with pleckstrin homology and src homology domains) protein. Tyrosine phosphorylation of these proteins creates recognition sites for both src homology ( $\mathrm{SH} 2$ ) binding and phosphotyrosine binding (PTB) domains of several downstream effector proteins. For example, tyrosine phosphorylation of the IRS proteins provides docking sites for $\mathrm{p} 85$, the regulatory subunit of the type 1A phosphatidylinositol (PI) 3-kinase, the protein tyrosine phosphatase SHP2, the Src family member kinase Fyn, and the small adapter proteins Grb2 and Nck.

Although the precise signaling function of each of these adapters/effectors remain poorly defined, each apparently serves distinct yet overlapping biological functions. For example, Grb2 appears to primarily function in the insulin regulation of Ras activation through the appropriate engagement of the Ras guanylnucleotide exchange factor, SOS. This pathway directly leads to extracellular signal-regulated kinase (ERK) activation (via Raf and MEK) and is an important cascade regulating several transcription events and eventually mitogenesis. On the other hand, there is well-documented evidence that Ras can interact with the catalytic subunit of the PI 3-kinase and modulate its activity (Avruch, 1998). The PI 3-kinase has been implicated in numerous biological responses, including the stimulation of transcription, mitogenesis, anti-apoptosis, protein synthesis, glycogen synthesis, and glucose transport (Shepherd et al., 1997). In addition, PI 3-kinase is involved in the regulation of the actin cytoskeleton, which is under the control of Rac and Rho family members. Thus, understanding the interrelationship between all these possible initial signaling events and the in vivo specificity 
required for a defined biological response remains a daunting but critical problem in cell biology and insulin action.

\section{Insulin-stimulated GLUT4 Vesicle Translocation}

One critical aspect of insulin action is the regulation of whole-body glucose homeostasis and peripheral tissue glucose uptake. This primarily results from the translocation of intracellular stored GLUT4 protein to the plasma membrane (Pessin et al., 1999; Rea and James, 1997). In the basal state, GLUT4 cycles slowly between the plasma membrane and one or more intracellular compartments, with the vast majority of the transporter residing within the cell interior. Activation of the insulin receptor triggers a large increase in the rate of GLUT4 vesicle exocytosis and a smaller decrease in the rate of internalization by endocytosis. The insulin-mediated increase in exocytosis is probably the major step for GLUT4 translocation, since a complete inhibition of GLUT4 endocytosis results only in a partial increase in plasma membrane-associated GLUT4 protein without affecting the extent of insulin-stimulated GLUT4 translocation. The overall insulin-dependent shift in the cellular dynamics of GLUT4 vesicle trafficking results in a net increase of GLUT4 protein levels on the cell surface, thereby increasing the rate of glucose uptake.

Substantial data have been accumulated demonstrating that the activation of the type IA PI 3-kinase and subsequent generation of $\mathrm{PI}(3,4,5) \mathrm{P} 3$ are essential for the insulin stimulation of GLUT4 translocation. For example, multiple studies using various pharmacological inhibitors, dominant-interfering mutants, expression of a phosphatidylinositol 5 ' phosphatase, and expression of a constitutively active catalytic subunit all are consistent with a necessary PI 3-kinase activity for insulin-stimulated GLUT4 translocation (reviewed in Shepherd et al., 1998). The formation of $3^{\prime}$ phosphoinositides activates the phosphoinositide-dependent protein kinase (PDK1). This phosphorylates another serine/threonine kinase Akt, also known as protein kinase $\mathrm{B}$ (PKB) or RAC-PK, on threonine 308. In addition, binding of PI-3,4,5-P3 to the amino-terminal pleckstrin homology $(\mathrm{PH})$ domain of Akt releases an inhibitory constraint, making it a more-efficient substrate for PDK1. Full activation of Akt also requires phosphorylation on serine 473 that may result from the activation of another putative kinase, PDK2, or perhaps through an alteration in substrate recognition of PDK 1 following threonine phoshorylation of Akt and/or via autophosphorylation (Balendran et al., 1999; Toker and Newton, 2000).

In any case, several studies have observed a direct correlation between Akt function and insulin-stimulated GLUT4 translocation (reviewed in Czech and Corvera, 1999). For example, stable expression of a constitutively active, membrane-bound form of Akt in 3T3L1 adipocytes results in increased glucose trans- 
port and persistent localization of GLUT4 to the plasma membrane. In addition, co-expression of an epitope-tagged GLUT4 with a dominant-interfering Akt mutant was reported to inhibit insulin-stimulated GLUT4 translocation. However, this issue appears to be substantially more complicated, as protein kinase $C \zeta$ (PKC $\zeta)$ also is activated by insulin through the formation of polyphosphoinositides in a wortmannin-sensitive (PI 3-kinase-dependent) manner. In addition, expression of $\mathrm{PKC} \zeta$ or PKC $\lambda$ (both atypical PKC family members) induces GLUT4 translocation (Kotani et al., 1998; Standaert et al., 1997). Expression of a dominant-interfering Akt mutant was found to inhibit insulin-stimulated protein synthesis without any significant effect on PKC $\lambda$ activation or GLUT4 translocation. Although expression of a dominant-interfering PKC $\lambda$ mutant had no effect on insulin-stimulated Akt activation, GLUT4 translocation was inhibited. It should also be noted that, in all these studies, the dominant-interfering PKC or Akt mutants resulted in only an approximate 50 percent reduction in insulinstimulated GLUT4 translocation. Thus, at present, there is no clear consensus for either Akt, PKC $\zeta$, and/or PKC $\lambda$ as essential downstream targets of PI 3-kinase activation mediating insulin-stimulated GLUT4 translocation (Figure 1).

Despite the absolute requirement for PI 3-kinase activation, it is also clear that insulin must generate additional signals that function in conjunction with the PI 3-kinase to stimulate GLUT4 translocation. For example, activation of PI 3-kinase by platelet-derived growth factor (PDGF), interleukin-4 (IL-4), or through engagement of the integrin receptors does not induce GLUT4 translocation in adipocytes (Guilherme and Czech, 1998; Isakoff et al., 1995; Staubs et al., 1998). In addition, two insulin receptor mutants have been identified that effectively undergo insulin-stimulated activation of the PI 3-kinase but are unable to induce GLUT4 translocation (Krook et al., 1997). These observations are not to be confused with studies in which expression of high levels of a constitutively active PI 3-kinase catalytic subunit alone induces GLUT4 translocation. In this situation, the PI 3-kinase is highly promiscuous, causing massive increases in various polyphosphoinositides, serine/threonine protein phosphorylation, and marked changes in cellular morphology. Therefore, it may activate GLUT4 translocation through a stress response or by circumventing the normal insulin regulatory pathways. In any case, the most-compelling evidence for a required additional PI 3-kinase-independent pathway was the use of a cell-permeable analog of $\mathrm{PI}(3,4,5) \mathrm{P} 3$ (Jiang et al., 1998). In these experiments, addition of the $\mathrm{PI}(3,4,5) \mathrm{P} 3$ analog had no effect on glucose uptake in the absence of insulin. As expected, treatment of cells with wortmannin (a PI 3-kinase inhibitor) prevented insulin-stimulated glucose transport, consistent with a requirement for the activation of the PI 3-kinase. However, treatment of adipocytes with wortmannin, insulin, plus the PI $(3,4,5) \mathrm{P} 3$ analog resulted in enhanced glucose uptake. Together, these data provide compelling evidence that, although the PI 3-kinase 


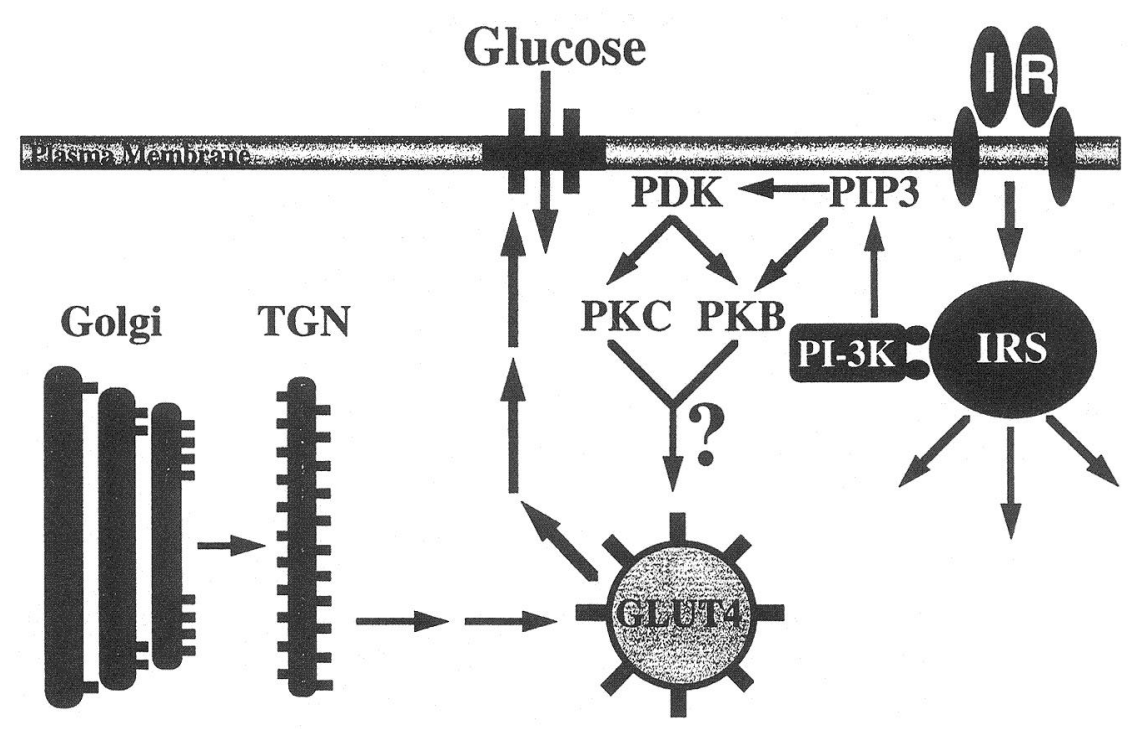

FIG. 1. Schematic model for the role of PI 3-kinase in insulin-stimulated GLUT4 translocation. Following GLUT4 protein synthesis in the endoplasmic reticulum and processing through the Golgi apparatus and the trans-Golgi network (TGN), the GLUT4 protein is localized to both tubulovesicular bodies and small cytosolic vesicles scattered throughout the cytoplasm. Activation of the insulin receptor induces the tyrosine phosphorylation of the IRS family of protein substrates that, in turn, engages the $\mathrm{SH} 2$ domains of the p85 PI 3-kinase regulator subunit. The activation and/or localization of the PI 3-kinase generates the formation of phosphatidylinositol $(3,4,5)$ trisphosphate (PIP3). PIP3 stimulates the kinase activity of phosphoinositide-dependent protein kinase (PDK) and interacts with protein kinase $\mathrm{B}(\mathrm{PKB})$ to make it a more-efficient substrate. In addition, PDK can phosphorylate and activate the atypical protein kinase $C$ (PKC) family members, zeta and lambda.

pathway is necessary, there is at least one additional insulin receptor-signaling pathway that functions independently of the PI 3-kinase.

What is the nature of this potential second signaling pathway? Several possibilities have been suggested, based upon the study of insulinomimetic agents that can stimulate glucose uptake and GLUT4 translocation without activating the insulin receptor. For example, exercise, hypoxia, and/or contraction in skeletal muscle induce GLUT4 translocation independently of PI 3-kinase activation (Goodyear and Kahn, 1998). It has been suggested that this results from activation of the adenosine monophosphate (AMP)-dependent protein kinase. In adipocytes, introduction of the nonhydrolyzable guanosine triphosphate(GTP) analogue $5^{\prime}-[\gamma$ thio]triphosphate (GTP $\gamma$ S) rapidly induces GLUT4 translocation by a PI 3-kinaseindependent mechanism (Elmendorf et al., 1998). More recently, several studies have suggested that the trimeric GTP-binding protein $(\mathrm{Gq} / \mathrm{G} 11) \alpha$ subunit may be 
required for insulin-stimulated GLUT4 translocation (Imamura et al., 1999; Kanzaki et al., 2000). Although controversial, the Gq/G11 stimulation of GLUT4 translocation was reported to function either downstream or in parallel with the PI 3-kinase. Thus, it is intriguing to speculate that the stimulatory action of GTP $\gamma \mathrm{S}$ may occur through activation of the Gq and/or G11 $\alpha$ subunits. However, since all these events appear to function independently of the insulin receptor, it is unlikely that they can account for the putative second pathway directly initiated by insulin.

A clue to this elusive PI 3-kinase-independent signal has emerged from a comparison of the proximal insulin receptor tyrosine phosphorylated substrates. As previously described, insulin stimulation results in the tyrosine phosphorylation of the IRS proteins, causing association and activation of the PI 3-kinase. Although many receptor and nonreceptor tyrosine kinases can phosphorylate $\mathrm{Cbl}$ in various cell types, only insulin is capable of tyrosine phosphorylating $\mathrm{Cbl}$ in adipocytes (Ribon and Saltiel, 1997). This appears to result from the presence of the $\mathrm{Cbl}$-associated adaptor protein (CAP) in adipocytes, which is required for insulin-stimulated $\mathrm{Cbl}$ tyrosine phosphorylation (Ribon et al., 1998). The CAP protein contains three adjacent carboxyl-terminal SH3 domains, with the last SH3 domain responsible for its association with the proline-rich domain of $\mathrm{Cbl}$. More recently, the amino-terminal domain of CAP was found to specifically interact with the caveolar protein flotillin. The insulin-stimulated, tyrosine-phosphorylated $\mathrm{Cbl}$ protein is localized to the lipid raft subdomains of the plasma membrane (Baumann et al., 2000). Importantly, expression of a dominant-interfering CAP mutant prevented the recruitment of tyrosine-phosphorylated $\mathrm{Cbl}$ to the lipid raft subdomains and inhibited insulin-stimulated glucose uptake and GLUT4 translocation. This occurred without any significant effect on the insulin stimulation of the PI 3-kinase or MAP kinase pathways. Since several studies have suggested that at least a portion of the insulin receptor is localized in caveolae, this may account for the unique ability of the insulin receptor to tyrosine phosphorylate $\mathrm{Cbl}$ in adipocytes via a CAP-dependent recruitment to the lipid raft subdomains (Figure 2). Obviously, additional studies are needed to connect the signaling events that occur downstream of $\mathrm{Cbl}$ and their relationship to the PI 3-kinase signaling pathway.

\section{GLUT4 Storage Compartments}

Irrespective of the specific signal transduction pathways required, they must necessarily impinge upon the intracellular GLUT4 storage compartments to induce their trafficking, docking, and fusion with the plasma membrane. Immunoelectron microscopy and subcellular fractionation studies have localized GLUT4 to several compartments of the recycling pathway, including the trans- 


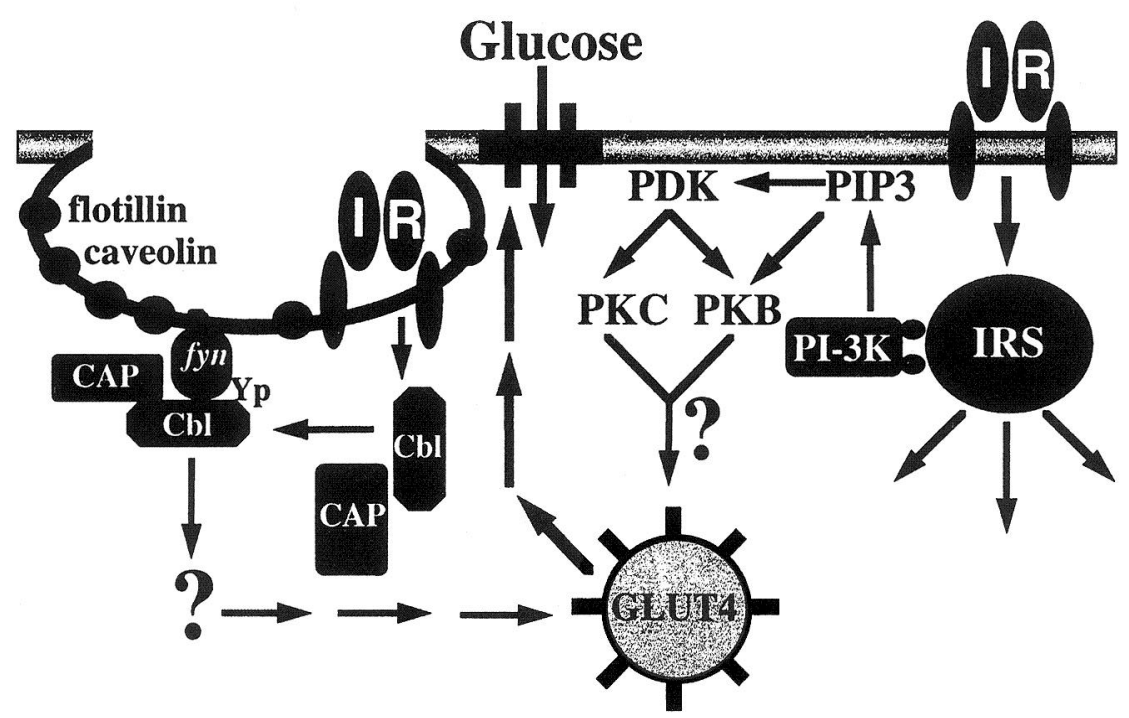

FIG. 2. Schematic model depicting the hypothesized role of the CAP/Cbl complex functioning in concert with the PI 3-kinase pathway in mediating insulin-stimulated GLUT4 translocation. In addition to the activation of the PI 3-kinase pathway, a portion of the cell surface insulin receptor is thought to reside within the flotillin/caveolin-enriched lipid raft domains. The insulin receptor can then tyrosine phoshorylate $\mathrm{Cbl}$ due to its localization (recruitment) to the lipid raft domains through the adaptor protein CAP.

Golgi network (TGN), clathrin-coated vesicles, and endosomes (Rea and James, 1997). However, the majority of GLUT4 appears to reside in tubulovesicular elements in the cytoplasm that lie beneath the plasma membrane and may represent specialized GLUT4 storage compartments. Several lines of evidence support this model. First, GLUT4 is targeted differently from GLUT1, which shows a markedly different steady-state distribution and localizes strongly to the plasma membrane under basal conditions. Second, compartment ablation studies using a horseradish peroxidase-transferrin receptor conjugate have shown that GLUT4 partitions into vesicular compartments that are largely distinct from those occupied by vesicle-associated membrane protein 3 (VAMP3) and transferrin receptor. Third, vesicle immunoabsorption studies have shown that, although some vesicle populations are enriched for both GLUT4 and endosomal markers, other vesicles appear to harbor a preponderance of GLUT4 and seem to exclude general endosomal markers.

These data support a model in which GLUT4 is partitioned into specialized, insulin-responsive storage compartments that represent the primary site of insulin action. One corollary of this model is that the GLUT4 protein must contain 
information that directs its localization to these specific compartments. Several groups have attempted to identify GLUT4 targeting motifs by expression of various mutant and chimeric GLUT4/GLUTI or GLUT4/transferrin receptor reporter constructs. A number of these early studies suggested that either the amino terminal FQQI motif and/or the carboxyl dileucine SLL motif was responsible for appropriate intracellular GLUT4 sequestration. Ilowever, subsequent analysis has demonstrated that these domains appear to function in the endocytosis of GLUT4. Thus, disruption of their function resulted in a default accumulation at the plasma membrane (Corvera et al., 1994; Garippa et al., 1996). Although a defined, intracellular sequestration sequence has not yet been identified, introduction of the GLUT4 carboxyl-terminal domain into adipocytes resulted in the spontaneous translocation of GLUT4 to the cell surface (Lee and Jung, 1997). Similarly, the insulin-responsive aminopeptidase (IRAP) co-localizes with GLUT4 and undergoes an insulin-stimulated plasma membrane translocation (Kandror and Pilch, 1994; Keller and Lienhard, 1994). Expression of the IRAP carboxyl terminus, which shares some sequence similarity with the GLUT4 carboxyl terminus, also caused GLUT4 translocation (Waters et al., 1997). Furthermore, insulin stimulation appears to unmask a cryptic antibody epitope at the carboxyl terminus (Smith et al., 1991; Wang et al., 1996). These data suggest that GLUT4 is sequestered away from the recycling endosome systems through the association of its carboxyl-terminal domain with a retention receptor. Competition with a related peptide releases the GLUT4-containing vesicles that can then enter the recycling endosome system and subsequently traffick to the plasma membrane. However, the identity of such a putative retention receptor has not been established and remains speculative. The lack of progress in this area leaves open the strong possibility that the elusive GLUT4-targeting motif may be scattered over noncontiguous residues that assemble into a discrete localization domain in the folded tertiary protein structure.

\section{Role of SNARE Proteins in GLUT4 Translocation}

Early studies examining intracellular transport processes suggested that regulated interactions between cognate receptor proteins displayed on the cytoplasmic faces of vesicle and target membranes mediate bilayer fusion events. The ability to reconstitute intercisternal Golgi transport in vitro provided the experimental means to identify the protein components of the vesicle fusion machinery. In this manner, two cytosolic proteins - the $\mathrm{N}$-ethylmaleimide (NEM)-sensitive fusion protein (NSF) and the soluble NSF attachment proteins (SNAPs) - were isolated (Calakos and Scheller, 1996). With the soluble components in hand, it became possible to search for their membrane-bound partners, termed SNARES for SNAP receptors. These studies, in combination with the characterization of highly purified synaptic vesicles, led to the identification of the target membrane SNAP 
receptors (t-SNAREs) as members of the syntaxin family and the vesicle membrane SNAP receptors (v-SNAREs) as members of the VAMP family. Subsequently, another target membrane SNARE, termed SNAP25 for synaptosomeassociated protein of $25 \mathrm{kDa}$, was found to form a stable complex with syntaxin. The fundamental importance of $\mathrm{v}$ - and t-SNAREs for synaptic vesicle exocytosis was demonstrated in neurons through the use of the tetanus and botulinum clostridial neurotoxins, which irreversibly block neurotransmitter release by selectively cleaving VAMP2, syntaxin 1, or SNAP-25 (Nieman et al., 1994). Subsequent studies using v- and t-SNARE partners reconstituted into separate liposome populations showed that interactions between cognate SNARE proteins form a bridge that brings vesicle pairs into sufficiently close apposition to cause bilayer mixing (Weber et al., 1998). Indeed, v- and t-SNARE partners may represent the minimal machinery for membrane fusion. Consistent with this hypothesis, structural studies have demonstrated that cognate $\mathrm{v}$ - and t-SNAREs form a parallel, four-helix bundle through coiled-coil domain interactions that may be sufficient to overcome the energetic barrier to bilayer fusion (Hanson et al., 1997; Sutton et al., 1998). In this model, the v-/t-SNARE complex forms first, allowing the subsequent binding of SNAPs, followed by NSF. The energy derived from ATP hydrolysis by NSF may serve to disassemble the very stable SNARE complex, freeing the SNAREs for another round of fusion (Figure 3).

Although in vitro binding studies have observed that $v$ - and t-SNAREs have the potential to interact promiscuously, under in vivo conditions, specific pairing of SNARE partners most likely provides one layer of specificity for membrane fusion events (Calakos et al., 1994; Scales et al., 2000). Consistent with this notion, both $\mathrm{v}$ and $\mathrm{t}$-SNAREs comprise large families of proteins that localize to discrete membrane compartments within the cell. SNAREs may thus help to demarcate membrane compartments with the potential to participate in the fusion process. Because their distribution within the cell may be critical for maintaining membrane compartment identity, several studies have investigated the mechanism by which SNAREs localize to specific membrane compartments. In the case of VAMP2, a specific signal within an $\alpha$-helical domain was found to specify localization to synaptic vesicles (Grote et al., 1995). More recently, two independent signals that cooperate to maintain syntaxin 6 in the TGN were identified: an $\alpha$-helical retention motif that traps syntaxin 6 in the TGN and a retrieval signal that returns wayward syntaxin 6 molecules back to the TGN (Watson and Pessin, 2000 ). These results are consistent with a model whereby the cell actively maintains the specific membrane compartment localization of SNARE proteins. Indeed, the fidelity of bilayer fusion events may be ensured at least in part by sequestering the fusogenic SNARE proteins in spatially segregated compartments. Maintaining compartment identity and fusion specificity is particularly important for GLUT4 and other protcins that navigate through multiple membrane compart- 


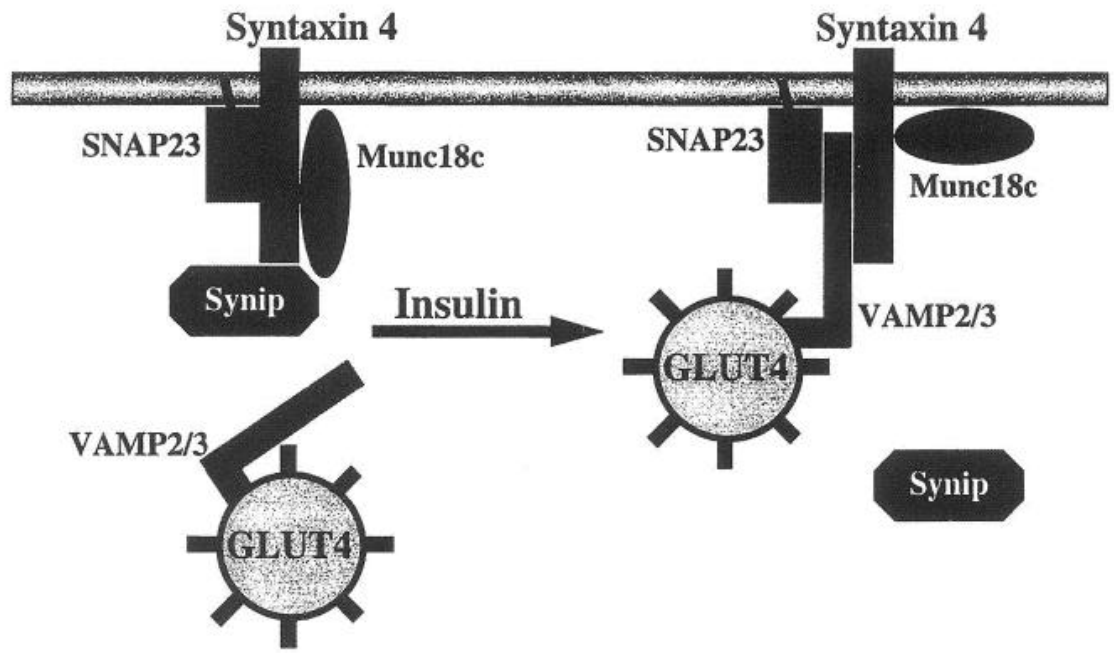

FIG. 3. SNARE model of GLUT4 vesicle docking with the plasma membrane. The intracellular GLUT4 vesicles also contain the v-SNARE proteins VAMP2 and/or VAMP3. The plasma membrane t-SNARE complex is composed of the transmembrane syntaxin 4 protein associated with the peripheral membrane protein, SNAP23. Moreover, the t-SNARE complex interacts with two additional proteins, Munc18c and Synip. Following insulin stimulation, the GLUT4 vesicles traffick to the plasma membrane, where a ternary complex involving VAMP2/3 and syntaxin 4/SNAP23 forms. Complex formation occurs concomitantly with the dissociation of the Synip protein, unmasking the syntaxin 4 coiled-coil domains, and through a conformation change in Munc18c.

ments during their biogenesis, intracellular storage, exocytosis, and retrieval from the plasma membrane. Although SNAREs play key roles during membrane fusion, several additional proteins help regulate the fusion process and may contribute to bilayer fusion specificity (Mayer, 1999; Pfeffer, 1999).

In the case of insulin-stimulated glucose transport in adipocytes, only syntaxin 4 has been implicated in GLUT4 vesicle trafficking. Inhibition of endogenous syntaxin 4 function by a variety of approaches - including overexpression of the cytosolic domain of syntaxin 4 or introduction of inhibitory syntaxin 4 antibodies - prevented insulin-stimulated GLUT4 translocation (reviewed in Pessin et al., 1999). Since the majority of syntaxin 4 is localized to the plasma membrane, GLUT4 vesicles presumably are prevented from docking or fusing with the cell surface when syntaxin 4 binding function is disrupted. Similarly, SNAP23 (a SNAP25 homolog in adipocytes) has been identified as a functional component of the t-SNARE complex and may contribute to GLUT4 vesicle fusion with the plasma membrane (Rea et al., 1998).

Two potential GLUT4 vesicle v-SNAREs, VAMP2 and VAMP3 (also known as cellubrevin), are expressed in adipocytes. Both of these isoforms share several 
characteristics expected of a GLUT4 v-SNARE protein. For example, both proteins partially co-localize in intracellular membranes with GLUT4 and translocate to the plasma membrane in response to insulin. Moreover, these two proteins can form stable complexes with syntaxin 4. Expression of either the VAMP2 or VAMP3 cytosolic domains inhibits GLUT4 translocation. Although these two proteins differ in their cytoplasmic amino-terminal regions, they differ by only one amino acid in their central t-SNARE-binding, coiled-coil domains. Based upon these close structural and physical properties between VAMP2 and VAMP3, a series of elegant cell and molecular approaches have been employed to distinguish the functional differences between these two isoforms. Initially, studies using relatively VAMP-specific proteases suggested that specific cleavage of VAMP2, but not VAMP3, reduced insulin-stimulated GLUT4 translocation (Cheatham et al., 1996). Consistent with a specific role for VAMP2, introduction of a short peptide corresponding to the unique amino-terminal extension of VAMP2 had a small effect on the appearance of GLUT4 at the plasma membrane (Rea et al., 1998). More importantly, in a series of specific compartment ablation studies, VAMP3 was found to be predominantly localized to the recycling endosome compartments defined by the transferrin receptor (Martin et al., 1996,1998; Millar et al., 1999b). These compartments contained approximately 50 percent of the GLUT4 protein but only a small fraction of the VAMP2-containing population. Ablation of the VAMP3/transferrin receptor endosomes had only a small effect on insulin-stimulated GLUT4 translocation but effectively inhibited GLUT4 translocation stimulated by GTPyS. These results support the notion that VAMP2 is the primary v-SNARE for insulin-stimulated translocation of GLUT4 vesicles to the plasma membrane. In contrast, VAMP3 appears to function in a different subpopulation of GLUT4 vesicles that are responsive to alternative signaling pathways.

\section{SNARE-associated Proteins in GLUT4 Translocation}

In addition to NSF, SNAPs, and SNAREs, several other proteins participate in the lipid bilayer fusion process. One critically important syntaxin-binding protein originally was identified in yeast as secl and subsquently as uncl8 in $\mathrm{C}$. elegans, ROP in D. melanogaster, and $\mathrm{nSecl}$ or Munc18a in mammals. The cytosolic $\mathrm{nSecl}$ protein binds with high affinity to syntaxin 1 and, at least in vitro, competes with the syntaxin 1 binding of VAMP2 and SNAP25, thereby preventing the formation of the syntaxin 1-VAMP2-SNAP25 ternary complex (Fujita $e t$ al., 1996; Pevsner et al., 1994). Consistent with a negative regulatory role for $\mathrm{nSec} 1$, increased ROP expression inhibited neurotransmitter release in vivo (Schulze et al., 1994). However, null or temperature-sensitive mutants of Secl homologues in S. cerevisiae, C. elegans, and D. melanogaster blocked secretion 
at specific steps, suggesting a positive role for Secl proteins during membrane fusion (Harrison et al., 1994; Hosono et al., 1992; Novick and Schekman, 1979). Recently, structural analysis of the nSecl-syntaxin 1 complex has been revealed and suggests that the nSecl maintains syntaxin 1 in a closed, inactive, conformational state, thereby preventing promiscuous membrane fusion (Misura et al., 2000 ). It is postulated that, upon activation, $\mathrm{nSec} 1$ could undergo a conformation change that leads to the open syntaxin 1 conformational state, thereby allowing membrane fusion. In this model, the function of $\mathrm{nSecl}$ would be to regulate the transition state between the open and closed syntaxin 1 conformations.

Since the original characterization of $\mathrm{nSec}$ 1/Munc18a as a neuronal-specific isoform, two additional ubiquitously expressed isoforms, termed Munc $18 \mathrm{~b}$ and Munc 18c, have been identified. Since only Munc $18 \mathrm{c}$ binds to syntaxin 4 with high affinity, substantial attention has focused on this isoform for its potential role in GLUT4 vesicle trafficking. We and others have shown that overexpression of Munc $18 \mathrm{c}$ inhibits GLUT4 vesicle translocation, perhaps by binding to syntaxin 4 and preventing its interaction with VAMP2 (Tellam et al., 1997; Thurmond et al., 1998,2000). Consistent with this view, Munc18c blocked the association of VAMP2 with syntaxin 4 in the yeast two-hybrid system and in in vitro binding experiments. However, although overexpression studies have yielded valuable information concerning the possible endogenous function of Munc18c, it is also important to examine Munc18c under physiological conditions, especially given the conflicting data and possible dual roles played by other Secl family members. To address this issue, we have used short peptides corresponding to conserved regions of Munc 18 isoforms to investigate the functional role of the syntaxin 4-Munc18c association in 3T3L1 adipocytes (Thurmond et al., 2000). In these studies, a short peptide corresponding to amino acids 459-483 of Munc18c blocked the ability of GLUT4 vesicles to integrate into the plasma membrane but did not block the movement of GLUT4 vesicles to the cell surface. Instead, GLUT4 vesicles were seen to accumulate beneath the plasma membrane, suggesting that an early stage of the fusion process was blocked. Since the Munc18c peptide disrupts the endogenous Munc18c-syntaxin 4 complex, these results suggest that native Munc18c plays a positive role during the fusion of GLUT4 vesicles with the plasma membrane, perhaps by maintaining syntaxin 4 in an optimal conformation for interactions with VAMP2. According to this model, Munc $18 \mathrm{c}$ binds to syntaxin 4 and inhibits GLUT4 vesicle fusion in the basal state, perhaps by keeping syntaxin 4 in the inactive, closed conformation. Upon insulin stimulation, Munc $18 \mathrm{c}$ may undergo a conformation change such that interactions between syntaxin 4 and VAMP2 are facilitated. When overexpressed, superphysiological doses of Munc18c may shift the equilibrium such that syntaxin 4 is maintained in a closed conformation. This leads to the observed inhibition of insulin-stimulated GLUT4 translocation under these conditions. Although this is an appealing model, there are currently no structural data regarding Munc18c/syn- 
taxin 4 binding or evidence that insulin can modulate these interactions in a timeframe consistent with GLUT4 translocation.

In ongoing efforts to identify additional regulatory components in GLUT4 translocation, we have isolated a novel syntaxin 4-binding protein termed synip (syntaxin 4-interacting protein). Synip was isolated using the yeast two-hybrid system with syntaxin 4 as "bait" and a 3T3L1 adipocyte cDNA expression library as a source for potential "prey" molecules (Min et al., 1999). Synip is predicted to contain several domains, including PDZ and EF hand domains at the amino terminus as well as two tandem coiled-coil domains and a WW motif at the carboxyl terminus. Synip interacts preferentially with syntaxin 4 (probably through the coiled-coil domains) and is expressed at high levels in adipose and skeletal muscle. Overexpression of wild-type synip had no effect on insulin-stimulated GLUT4 vesicle translocation. However, the carboxyl-terminal half of synip (which includes the coiled-coil and WW domains) strongly inhibited the ability of insulin to stimulate GLUT4 translocation when overexpressed. In addition, insulin stimulation resulted in the dissociation of full-length synip from syntaxin 4. These results suggest that the carboxyl-terminal region binds to syntaxin 4 and that the amino-terminal region provides the insulin regulatory sites required for the dissociation of the synip-syntaxin 4 complex. Thus, synip may function as a fusion control switch at the plasma membrane. In the basal state, the binding of synip effectively masks the syntaxin 4 molecule and prevents nonspecific vesicle fusion. In this model, insulin stimulation would result in the release of synip, thereby exposing the syntaxin 4 coiled-coil domains, making them available for interactions with VAMP2 (Figure 3). Obviously, further experimentation is needed to test this model. We currently are investigating the upstream signaling mechanism used by insulin to cause the dissociation of the synip-syntaxin 4 complex.

\section{Role of Small GTP Binding Proteins in GLUT4 Vesicle Translocation}

The large family of small GTP binding proteins play essential roles in many cell biological processes, including receptor tyrosine kinase signal transduction and intracellular vesicle trafficking. Rab proteins comprise the largest branch of the Ras superfamily of small GTPases, with over 50 mammalian isoforms known. In contrast to the SNARE proteins that are central in catalyzing the bilayer fusion event, Rab GTPases appear to function in upstream processes such as the initial tethering or docking of vesicles with their target membranes (Brennwald, 2000). Consistent with this proposed function, Rabs localize to the cytoplasmic faces of all organelles involved in membrane transport. This process of membrane delivery requires the cycling of the Rab proteins between the guanosine diphosphate (GDP)- and GTP-bound states. For example, the delivery of cargo vesicles requires GTP-bound Rab followed by GTP hydrolysis. Subsequently, the GDP- 
bound Rab is extracted from the target membrane by guanylnucleotide dissociation inhibitor (GDI) proteins and recycled back to the donor membrane for another round of vesicle transport.

Although insulin-responsive cells express many Rab isoforms, Rab4 has been specifically implicated in the insulin regulation of GLUT4 exocytosis (Cormont et al., 1993,1996; Mora et al., 1997; Shibata et al., 1996). Initially, Rab4 was found to co-localize with the GLUT4-enriched, low-density microsome fraction of adipocytes. In addition, insulin stimulation resulted in the redistribution of Rab4 from the microsome fraction to the cytosolic fraction, in parallel with the movement of GLUT4 to the plasma membrane. Moreover, introduction of a Rab4 carboxyl-terminal peptide or expression of a carboxyl-terminal Rab4 truncation mutant inhibited insulin-stimulated GLUT4 translocation (Knight et al., 2000; Shibata et al., 1996). Finally, overexpression of wild-type Rab4 inhibited GLUT4 translocation, presumably by preventing correct cycling of the GTPase (Mora et al., 1997). Together, these data implicate Rab4 as a possible mediator of fusion events related to GLUT4 trafficking to the cell surface. Although these data provide suggestive evidence for an important functional role of Rab4, there is no data indicating whether or not insulin modulates Rab4 GDP/GTP cycling or the specific Rab4 effectors required for GLUT4 vesicle translocation.

Another important Ras subfamily of small, GTP binding proteins involved in vesicle trafficking is the ADP-ribosylation factors, ARFs (Chavrier and Goud, 1999). ARFs are thought to function during coat protein recruitment in the early stages of vesicle budding. In the GDP-bound state, ARFs are cytosolic; however, upon GTP loading, they associate with membranes and recruit cytosolic coat components to the donor membrane compartment. The coat proteins then deform the donor membrane into a bud and bind cargo molecules. Given their widespread roles in membrane-trafficking processes, it seems likely that one or more ARF isoforms participate in various stages of GLUT4 vesicle trafficking. ARF6 has been implicated in GLUT4 vesicle trafficking. Introduction of myristoylated peptides corresponding to the amino terminus of ARF6 was reported to inhibit GLUT4 vesicle translocation by about 50 percent in permeabilized adipocytes (Millar et al., 1999a). In contrast, myristoylated peptides from other ARF isoforms did not affect GLUT4 translocation. ARF6 is intriguing because experiments in fibroblasts have suggested that ARF6 links membrane trafficking with the organization of the actin cytoskeleton. Agents that disrupt actin organization also inhibit GLUT4 translocation (Omata et al., 2000; Radhakrishna et al., 1999). However, other studies using a dominant-interfering ARF6 mutant found no significant effect on basal or insulin-stimulated glucose transport (Yang and Mueckler, 1999). Thus, although ARF isoforms are likely to participate in GLUT4 vesicle trafficking, additional work is needed to refine our understanding of the potential role played by ARF6 and perhaps other ARF isoforms. 


\section{GLUT4 Endocytosis}

Although it is well documented that the major effect of insulin is to enhance the rate of GLUT4 exocytosis, insulin also decreases the rate of GLUT4 endocytosis approximately two to three fold (Czech, 1995; Holman and Cushman, 1994; Kandror and Pilch, 1996). Indeed, a complete understanding of the molecular mechanism underlying GLUT4 endocytosis could afford opportunities to develop novel drug therapies aimed at slowing the rate of GLUT4 internalization, thus enhancing glucose uptake under diabetic conditions. Currently, we only have a vague understanding of the molecular mechanism responsible for GLUT4 internalization. Several lines of evidence suggest that GLUT4 endocytosis primarily occurs through clathrin-coated pits. For example, GLUT4 has been localized to clathrin-coated pits by immunofluorescence and electron microscopy studies. Maneuvers that inhibit clathrin-mediated endocytosis prevent GLUT4 internalization (Garippa et al., 1996; Nishimura et al., 1993; Robinson et al., 1992; Slot et al., 1991a,1991b).

The mechanism by which clathrin-coated pits invaginate and form free vesicles is unclear; however, the cytosolic GTPase dynamin appears to play a key role in this process. Dynamin is a $100-\mathrm{kDa}$ protein with an amino-terminal GTPase domain, a central pleckstrin homology domain, and a carboxyl-terminal proline-rich region. Dynamin wraps around the necks of invaginating vesicles as a spiral. This process has been visualized in seminal experiments in Drosophila, where temperature-sensitive mutants of the dynamin homologue shibire yield a paralytic phenotype at the nonpermissive temperature. Electron microscopy analysis of the presynaptic termini of these flies revealed an absence of synaptic vesicles and an abundance of clathrin-coated pits with electron-dense collars around their necks, suggesting that a late stage of endocytosis was blocked (Kosaka and Ikeda, 1983). The shibire temperature-sensitive mutations occur near the GTPase domain and, together with experiments using GTP $\gamma \mathrm{S}$, it was proposed that GTPase activity of dynamin was necessary for the scission process. In this model, the energy derived from GTP hydrolysis induces a conformational change in dynamin such that the necks of invaginating vesicles are constricted to the point where scission occurs (McNiven, 1998). However, this model recently has been challenged. GTP hydrolysis may instead cause the elongation of dynamin spirals, resulting in stretching and eventual scission of the vesicle neck (McNiven et al., 2000). Alternatively, recent work suggests that dynamin may function as a classical GTPase molecular switch that recruits other effector molecules (e.g., endophilin), which then participate in the formation of coated vesicles (Ringstad et al., 1999).

Although the specific details of its molecular mechanism remain controversial, it is clear the dynamin plays an essential role in GLUT4 endocytosis. Multiple studies expressing dominant-interfering dynamin mutants or specific peptides to disrupt dynamin binding to other essential effectors resulted in a near-complete 
inhibition of GLUT4 endocytosis (Al-Hasani et al., 1998; Kao et al., 1998; Volchuk et al., 1998). However, a potential mechanism by which insulin might regulate dynamin function has not been forthcoming. One possibility is that the proline-rich domain of dynamin is well known to associate with various effector SH3 domains that, in turn, enhance the dynamin GTPase activity (Muhlberg et al., 1997). In this manner, Grb2 has been reported to associate with dynamin and induce the binding of the dynamin-Grb2 complex to tyrosine phosphorylated Shc and IRS1 (Ando et al., 1994). These results suggest that the formation of this higher-order complex either inhibits dynamin activity and/or results in the sequestration of dynamin away from the GLUT4-containing, clathrin-coated pits. Alternatively, a recent study reported that insulin induces the tyrosine phosphorylation of dynamin (Baron et al., 1998). In either case, the functional role of these events remains to be determined, as there is currently no evidence that insulin modulates dynamin localization, GTPase activity, or pinchase function in vivo.

\section{Summary and Future Prospects}

Since the discovery of insulin in the 1920 s, it took another 60 years to make the seminal observation that insulin stimulates glucose uptake by inducing the translocation of glucose transporter proteins from intracellular storage sites to the plasma membrane. During the next 20 years, researchers have identified multiple family members of the facilitative glucose transporter and demonstrated that the GLUT4 isoform is the predominant insulin-responsive transporter in striated muscle and adipose tissue. This protein is localized to at least two distinct intracellular compartments, one that is apparently similar to the general recycling endosome system and a second, more-specialized insulin-responsive compartment. The overall translocation process is similar to that utilized during synaptic transmission. It requires the specific pairing of GLUT4 vesicle v-SNAREs with plasma membrane t-SNAREs. Future studies are needed to identify the specific events and regulatory steps involved in the trafficking, docking, and fusion of these intracellular GLUT4 compartments with the plasma membrane.

In addition to the important role of intracellular GLUT4 localization, it is becoming increasingly apparent that insulin signaling is compartmentalized. We now know that insulin activation of PI 3-kinase and generation of phosphatidylinositol-3,4,5-trisphosphate is necessary for insulin-stimulated glucose uptake and GLUT4 translocation. More-recent studies indicate that this pathway is not sufficient and that a second insulin-stimulated pathway functions in concert with the PI 3-kinase. This pathway is also compartmentalized and utilizes the insulinstimulated recruitment of tyrosine-phosphorylated $\mathrm{CAP} / \mathrm{Cbl}$ complex to the flotillin-enriched lipid raft plasma membrane subdomains. Thus, understanding the subsequent downstream events from both the PI 3-kinase and the CAP/Cbl complex are critical issues that require substantial investigation. Moreover, the 
integration of these signals and their specific roles in modulating t-SNARE and v-SNARE interactions will be a fruitful area to pursue. With an improved molecular understanding of these regulatory events, the prospects for the rational development of specifically targeted antidiabetic drugs will become a viable possibility.

\section{REFERENCES}

Al-Hasani, H., Hinck, C.S., and Cushman, S.W. (1998). J. Biol. Chem. 273, 17504-17510.

Ando, A., Yonezawa, K., Gout, I., Nakata, T., Ueda, H., Hara, K., Kitamura, Y., Noda, Y., Takenawa, T., Hirokawa, N. (1994). EMBO J. 13, 3033-3038.

Avruch, J. (1998). Mol. Cell. Biochem. 182, 31-48.

Balendran, A., Casamayor, A., Deak, M., Paterson, A., Gaffney, P., Currie, R., Downes, C.P., and Alessi, D.R. (1999). Curr. Biol. 22, 393-404

Baron, V., Alengrin, F., and Van Obberghen, E. (1998). Endocrinology 139, 3034-3037.

Baumann, C.A., Ribon, V., Kanzaki, K., Thurmond, D.C., Mora, S., Shigematsu, S., Bickel, P.E., Pessin, J.E., and Saltiel, A.R. (2000). Nature 407, 202-207.

Brennwald, P. (2000). J. Cell. Biol. 149, 1-4.

Calakos, N., and Scheller, R.H. (1996). Physiol. Rev. 76, 1-29.

Calakos, N., Bennett, M.K., and Peterson, K.E. (1994). Science 263, 1146-1149.

Chavrier, P., and Goud, B. (1999). Curr. Opin. Cell Biol. 11, 466-475.

Cheatham, B., Volchuk, A., Kahn, C.R., Wang, L., Rhodes, C.J., and Klip, A. (1996). Proc. Natl. Acad. Sci. U.S.A. 93, 15169.15173.

Cormont, M., Tanti, J.F., Zahraoui, A., Vanobberghen, E., Tavitian, A., and Le Marchand-Brustel, Y. (1993). J. Biol. Chem. 268, $19491-19497$.

Cormont, M., Bortoluzzi, M. N., Gautier, N., Mari, M., Van Obberghen, E., and Le Marchand-Brustel, Y. (1996). Mol. Cell. Biol. 16, 6879-6886.

Corvera, S., Chawla, A., Chakrabarti, R., Joly, M., Buxton, J., and Czech, M.P. (1994). J. Cell. Biol. 126, 979-989

Czech, M.P. (1995). Annu. Rev. Nutrit. 15, 441-471.

Czech, M.P., and Corvera, S. (1999). J. Biol. Chem. 274, 1865-1868.

Elmendorf, J.S., Chen, D., and Pessin, J.E. (1998). J. Biol. Chem. 273, 13289-13296.

Fujita, Y., Sasaki, T., Fukui, K., Kotani, H., Kimura, T., Hata, Y., Sudhof, T.C., Scheller, R.H., and Takai, Y. (1996). J. Biol. Chem. 271, 7265-7268.

Garippa, R.J., Johnson, A., Park, J., Petrush, R.L., and McGraw, T.E. (1996). J. Biol. Chem. 271, 20660-20668.

Goodyear, L.J., and Kahn, B.B. (1998). Annu. Rev. Med. 49, 235-261.

Grote, E., Hao, J.C., Bennett, M.K., and Kelly, R.B. (1995). Cell 81, 581-589.

Guilherme, A., and Czech, M.P. (1998). J. Biol. Chem. 273, 33119-33122.

Hanson, P.I., Roth, R., Morisaki, H., Jahn, R, and Heuser, J.E. (1997). Cell 90, 523-535.

Harrison, S.D., Broadie, K., van de Goor, J., and Rubiı, G.M. (1994). Neurun 13, 555-566.

Holman, G.D., and Cushman, S.W. (1994). Bioessays 16, 753-759.

Hosono, R., Hekimi, S., Kamuya, Y., Sassa, T., Murakami, S., Nishiwaki, K., Miwa, J., Taketo, A., and Kodaira, K.I. (1992). J. Neurochem. 58, 1517-1525.

Imamura, T., Vollenweider, P., Egawa, K., Clodi, M., Ishibashi, K., Nakashima, N., Ugi, S., Adams, J.W., Brown, J.H., and Olefsky, J.M. (1999). Mol. Biol. Cell 19, 6765-6774.

Isakoff, S.J., Taha, C., Rose, E., Marcusohn, J., Klip, A., and Skolnik, E.Y. (1995). Proc. Natl. Acad. Sci. U.S.A. 92, 10247-10251. 
Jiang, T., Sweeney, G., Rudolf, M.T., Klip, A., Traynor-Kaplan, A., and Tsien, R.Y. (1998). J. Biol. Chem. 273, 11017-11024.

Kandror, K., and Pilch, P.F. (1994). J. Biol. Chem. 269, 138-142.

Kandror, K.V., and Pilch, P.F. (1996). Am. J. Physiol. 271, E1-E14.

Kanzaki, M., Watson, R.T., Artemyev, N.O., and Pessin, J.E. (2000). J. Biol. Chem. 275, 7167-7175.

Kao, A.W., Ceresa, B.P., Santeler, S.R., and Pessin, J.E. (1998). J. Biol. Chem. 273, 25450-25457.

Keller, S.R., and Lienhard, G.E. (1994). Trends Cell Biol. 4, 115-119.

Knight, J.B., Cao, K.T., Gibson, G.V., and Olson, A.L. (2000). Endocrinology 141, 208-218.

Kosaka, T., and Ikeda, K. (1983). J. Neurobiology 14, 207-225.

Kotani, K., Ogawa, W., Matsumoto, M., Kitamura, T., Sakaue, H., Hino, Y., Miyake, K., Sano, W., Akimoto, K., Ohno, S., and Kasuga, M. (1998). Mol. Cell. Biol. 18, 6971-6982.

Krook, A., Whitehead, J.P., Dobson, S.P., Griffiths, M.R., Ouwens, M., Baker, C., Hayward, A.C., Sen, S.K., Maassen, J.A., Siddle, K., et al. (1997). J. Biol. Chem. 272, 30208-30214.

Lee, J.S., and Pilch, P.F. (1994). Am. J. Physiol. 266, C319-C334.

Lee, W., and Jung, C.Y. (1997). J. Biol. Chem. 272, 21427-21431.

Martin, L.B., Shewan, A., Millar, C.A., Gould, G.W., and James, D.E. (1998). J. Biol. Chem. 273, 1444-1452.

Martin, S., Tellam, J., Livingstone, C., Slot, J.W., Gould, G.W., and James, D.E. (1996). J. Cell. Biol. $134,625-635$.

Mayer, A. (1999). Curr. Opin. Cell Biol. 11, 447-452.

McNiven, M.A. (1998). Cell 94, 151-154

McNiven, M.A., Cao, H., Pitts, K.R., and Yoon, Y. (2000). Trends Biochem. Sci. 25, 115-120.

Millar, C.A., Powell, K.A., Hickson, G.R., Bader, M.F., and Gould, G.W. (1999a). J. Biol. Chem. 274, 17619-17625.

Millar, C.A., Shewan, A., Hickson, G.R., James, D.E., and Gould, G.W. (1999b). Mol. Biol. Cell 10, 3675-3688.

Min, J., Okada, S., Coker, K., Ceresa, B.P., Elmendorf, J.S., Syu, L.-J., Noda, Y., Saltiel, A.R., and Pessin, J.E. (1999). Mol. Cell 3, 751-760.

Misura, K.M., Scheller, R.H., and Weis, W.I. (2000). Nature 404, 355-362.

Mora, S., Monden, I., Zorzano, A., and Keller, K. (1997). Biochem. J. 324, 455-459.

Muhlberg, A.B., Warnock, D.E., and Schmid, S.L. (1997). EMBO J. 16, 6676-6683.

Nieman, H., Blasi, J., and Jahn, R. (1994). Trends. Cell Biol. 4, 179-185.

Nishimura, H., Zarnowski, M.J., and Simpson, I.A. (1993). J. Biol. Chem. 268, 19246-19253.

Novick, P., and Schekman, R. (1979). Proc. Natl. Acad. Sci. U.S.A. 76, 1858-1862.

Omata, W., Shibata, H., Li, L., Takata, K., and Kojima, I. (2000). Biochem. J. 346(pt. 2), 321-328.

Pessin, J., Thurmond, D., Elmendorf, J., Coker, K., and Okada, S. (1999). J. Biol. Chem. 274, 2593-2596.

Pevsner, J., Hsu, S.-C., and Scheller, R.H. (1994). Proc. Natl. Acad. Sci. U.S.A. 91, 1445-1449.

Pfeffer, S.R. (1999). Nature Cell Biol. 1, E17-E22.

Radhakrishna, H., Al-Awar, O., Khachikian, Z., and Donaldson, J.G. (1999). J. Cell. Sci. 112, 855-866.

Rea, S., and James, D. (1997). Diabetes 46, 1667-1677.

Rea, S., Martin, L.B., McIntosh, S., Macaulay, S.L., Ramsdale, T., Baldini, G., and James, D.E. (1998). J. Biol. Chem. 273, 18784-18792.

Ribon, V., and Saltiel, A.R. (1997). Biochem. J. 324, 839.

Ribon, V., Printen, J.A., Hoffman, N.G., Kay, B.K., and Saltiel, A.R. (1998). Mol. Cell. Biol. 18, 872-879.

Ringstad, N., Gad, II., Low, P., Di Paolo, G., Brodin, L., Shupliakov, O., and De Camilli, P. (1999). Neuron 24, 143-154.

Robinson, L.J., Pang, S., Harris, D.S., Heuser, J., and James, D.E. (1992). J. Cell. Biol. 117, 1181-1196.

Scales, S.J., Chen, Y.A., Yoo, B.Y., Patel, S.M., Doung, Y.-C., and Scheller, R.H. (2000). Neuron 26, 457-464. 
Schulze, K.L., Littleton, J.T., Salzberg, A., Halachmi, N., Stern, M., Lev, Z., and Bellen, H.J. (1994). Neuron 13, 1099-1108.

Shepherd, P.R., Siddle, K., and Nave, B.T. (1997). Biochem. Soc. Trans. 25, 978-981.

Shepherd, P.R., Withers, D.J, and Siddle, K. (1998). Biochem. J. 333, 471-490.

Shibata, H., Omata, W., Suzuki, Y., Tanaka, S., and Kojima, I. (1996). J. Biol. Chem. 271, 9704-9709.

Slot, J.W., Geuze, H.J., Gigengack, S., James, D.E., and Lienhard, G.E. (1991a). Proc. Natl. Acad. Sci. U.S.A. 88, 7815-7819.

Slot, J.W., Geuze, H.J., Gigengack, S., Lienhard, G.E., and James, D.E. (1991b). J. Cell. Biot. 113, 123-135.

Smith, R.M., Charron, M.J., Shah, N., Lodish, H.F., and Jarett, L. (1991). Proc. Natl. Acad. Sci. U.S.A. 88, 6893-6897.

Standaert, M.L., Galloway, L., Karnam, P., Bandyopadhyay, G., Moscat, J., and Farese, R.V. (1997). J. Biol. Chem. 272, 30075-30082.

Staubs, P.A., Nelson, J.G., Reichart, D.R., and Olefsky, I.M. (1998). J. Biol. Chem. 273, 25139-25147.

Sutton, R., Fasshauer, D., Jahn, R, and Brunger, A. (1998). Nature 395, 347-353.

Tellam, J.T., Macaulay, S.L., Mcintosh, S., Hewish, D.R., Ward, C.W., and James, D.E. (1997). J. Biol. Chem. 272, 6179-6186.

Thurmond, D.C., Ceresa, B.P., Okada, S., Elmendorf, J.S., Coker, K., and Pessin, J.E. (1998). J. Biol. Chem. 273, 33876-33883.

Thurmond, D.C., Kanzaki, M., Khan, A.H., and Pessin, J.E. (2000). Mol. Cell. Biol. 20, 379-388.

Toker, A, and Newton, A.C. (2000). .J. Biol. Chem. 275, 8271-8274.

Volchuk, A., Narine, S., Foster, L.J., Grabs, D., De Camilli, P., and Klip, A. (1998). J. Biol. Chem. 273, $8169-8176$.

Wang, W., Hansen, P.A., Marshall, B.A., Holloszy, J.O., and Mueckler, M. (1996). J. Cell. Biol. 135, 415-430.

Waters, S. B., D'Auria, M., Martin, S.S., Nguyen, C., Kozma, L.M., and Luskey, K.L. (1997). J. Biol. Chem. 272, 23323-23327.

Watson, R.T., and Pessin, J.E. (2000), J. Biol. Chem. 275, 1261-1268.

Weber, T., Zemelman, B. V., McNew, J.A., Westermann, B., Gmachl, M., Parlati, F., Sollner, T.H., and Rothman, J.E. (1998). Cell 92, 759-772.

Yang, C.Z., and Mueckler, M. (1999). J. Biol. Chem. 274, 25297-25300. 
